\title{
晚二叠世磁性地层及国际对比意义
}

\section{金玉玕尚庆华 曹长群}

(中国科学院南京地质古生物研究所, 南京 210008)

摘要 磁性地层与化石带及同位素年龄的检验结果表明: 石炭纪二叠纪反极性超带与二叠 纪-三叠纪混合极性超带的分界—伊拉瓦拉极性反转出现在瓜德鲁普世沃德期末, 同位素年 龄为 $265 \mathrm{Ma}$. 晚二叠世极性地层可以划分为 4 个极性带, 分别约相当瓜德鲁普世卡匹敦期、乐 平世吴家坪期、长兴期早期和长兴期晚期. 这一磁性地层序列为超越长期未能克服的沉积相 和生物地理隔障, 为建立高精度的晚二叠世地层对比提供了依据.

\section{关键词 二叠纪 磁性地层 生物地层 伊拉瓦拉极性反转}

地磁极性面和地磁极性地层单位具有世界性分布, 而且基本上是等时的. 二叠纪生物区 系的分异极为强烈, 很难依靠生物地层建立全球性对比, 所以磁性地层资料显得特别重要.二 叠纪早期和中期(约 $30 \mathrm{Ma}$ ) 都属于石炭纪二叠纪反极性超带( CPRM) 或基亚曼(Kiaman) 极性 期, 只有晚期(约 $15 \mathrm{Ma}$ ) 属于二叠纪-三叠纪混合极性超带( PTMM) 或伊拉瓦拉( Illawarra) 极性 期 ${ }^{[1,2]}$. 两个超带的界面以第一个正极性带的出现为标志, 称伊拉瓦拉极性反转( Illawarra Reversal), 是各大陆都能识别的时间标志. 在伊拉瓦拉极性反转之上的瓜德鲁普世和乐平世地 层中, 正反极性层交替出现, 有利于建立详细的磁性地层分带, 作为跨地区高精度对比的重要 依据. 我国具备层序完整的晚二叠世海相和陆相地层, 近 10 年来成为国际合作研究二叠纪三叠纪混合极性超带的重点地区, 并建立了一些磁性地层剖面. 地磁极性缺乏个性, 如果没有 生物地层和同位素年龄资料佐证, 时代很难确定. 虽然目前建立的磁性地层剖面的古地磁测 试结果被认为可信度较高, 但是大都没有充分利用生物地层资料进行校验, 以致各家对极性层 年代的解释和对比很不一致. 例如, 李华梅等人 ${ }^{[3]}$ 确定茅口组顶部为反极性, 长兴组包含正极 性带, 推测伊拉瓦拉极性反转面出现在吴家坪组. 另一方面, Embleton 等人 ${ }^{[4]}$ 认为上石盒子组 下部的正极性层代表伊拉瓦拉极性反转, 时代为 Ufimian 期或瓜德鲁普世(Guadalupian) 的早 期. 吴家坪阶和 Ufimian 阶下界的数值年龄分别约为 $258 \mathrm{Ma}$ 和 $272 \mathrm{Ma}$ ，两者相差的时限超过 $10 \mathrm{Ma}$. 作者等根据最新的生物地层和同位素年龄资料, 重新分析各极性层的地质时代后, 发 现各磁性地层剖面的对应性很高, 足以建立一个晚二叠世综合磁性地层序列. 现将根据生物 地层、同位素年龄和磁性地层互相校验而建立的综合层序及其国际对比潜力评述如下.

\section{1 伊拉瓦拉极性反转事件}

伊拉瓦拉极性反转事件首先在澳大利亚悉尼盆地南部确定. Irving 等人 ${ }^{[5]}$ 发现二叠纪岩 层的天然剩磁大部分呈现反极性, 从 Gerringong 火山岩之上开始出现正极性, 并形成正、反极 性层交替的序列(图 1)。 以后在俄罗斯莫斯科盆地 Tatar 组下部 ${ }^{[6]}$ 、美国瓜德鲁普山的 Yatse 组 ${ }^{[7]}$ 、德国 Thuringen 盆地赤底统 Havel 组 ${ }^{[8]}$ 等地发现, 使这些处在不同大陆并缺乏可资对比的 化石的地层, 有可能用一条等时线联结起来, 从而引起广泛的注意. 不过, 对伊拉瓦拉极性反 转的层位一直缺乏精确的生物地层控制, 与海相化石带的层位关系尤不明确. 


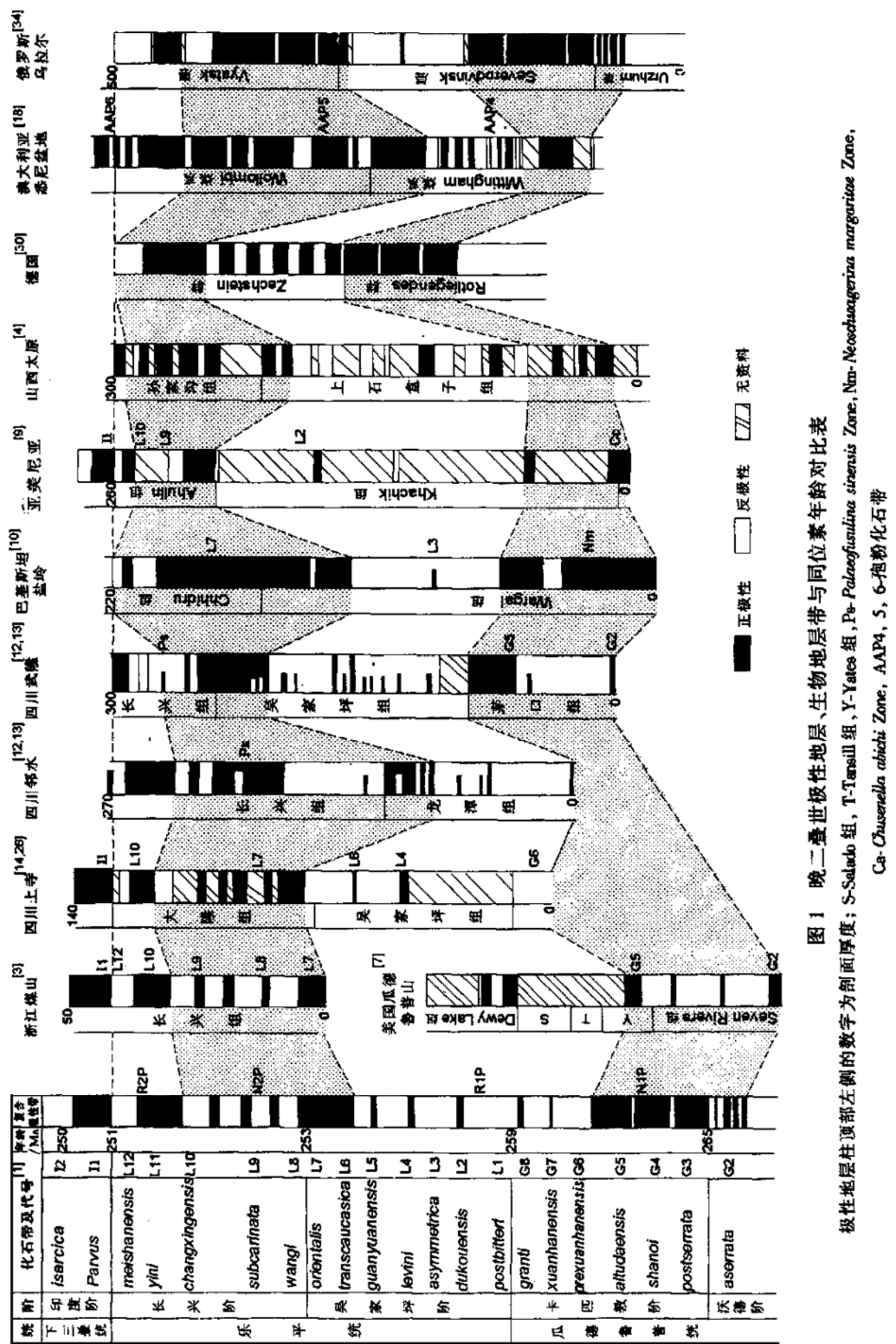




\section{1 在海相沉积区的层位}

晚二叠世海相地层的化石分带较详细, 便于确定伊拉瓦拉极性反转的详细层位. 目前已 有生物地层资料控制较好, 古地磁测试结果的可信度较高的几个剖面可供分析. 在瓜德鲁普 统的标准地点一美国新墨西哥州的瓜德鲁普山的礁后相序列中, 早期的研究者发现正极性 层出现在相当于 Bell Canyon 组上部的 Yatse 组 ${ }^{[7]}$. 最近系统研究该地盆地相序列的结果, 表明 伊拉瓦拉极性反转的层位为 Bell Canyon 组的 Hegler 灰岩段与 Pinery 灰岩段之间, 相当沃德阶 (Wordian Stage) 的顶部, 即 J. aserrata 带顶部. 同时, Bowring 等人 ${ }^{[16]}$ 采用锆石铀-铅同位素稀 释热电离子质谱分析法, 测得 Hegler 灰岩段顶部的凝灰岩层年龄为( 265.3 \pm 0.2 ) Ma.

在亚美尼亚, 晚二叠世最低的正极性层出现在 Arpin 组 Chusenella abichi 带顶部, 相当 Neoschwagerina margaritae 带之顶 ${ }^{[9]}$; 在巴基斯坦盐岭则见于 Wargal 组下部, 该部也产 $N$. cf. margaritae $^{[10,11]}$. 在我国, 陈海泓等人 ${ }^{[12]}$ 确定四川武隆县江口茅口组顶部(相当第三段底部) 出现正极性带. 研究者 ${ }^{[13,14]}$ 曾怀疑该带并不代表伊拉瓦拉极性反转事件,而采用龙潭组的正 极性带为其代表, 认为二叠纪 三叠纪混合极性超带起始于吴家坪阶上部, 而将茅口组上部的 正极性带归入 CPRM. 显然, 这一推断与已知的生物地层资料相矛盾. 因为, 在四川武隆县江 口剖面, 层位最低的正向带出现在离茅口组顶面 $90 \mathrm{~m}$ 处, 接近硅质层段之底, 低于 Yabeina 带. 如与宣汉渡口剖面对比 ${ }^{[15]}$, 此层应处在 Jinogondolella aserrata 带上部, 大体相当 $N$. margaritae 带, 也即沃德阶顶部.

总之, 在海相地层测得的磁性地层资料充分说明, 伊拉瓦拉极性反转的层位为沃德阶顶 部, 相当筳类 $N$. margaritae 带, 牙形化石 $J$. aserrata 带的顶部, 同位素年龄约为 $265 \mathrm{Ma}$.

\section{2 在命名地区的层位}

在伊拉瓦拉极性反转的命名地一澳大利亚新南威尔士州的 Kiaman 地区, 晚二叠世生物 地层序列虽然与瓜德鲁普统和乐平统的标准序列难以直接对比, 但可以通过巴基斯坦盐岭等 过渡性地区的晚二叠世生物地层进行间接对比. Gerrington 火山岩层之上的 Illawarra 煤系所产 的孢粉化石被归入 $\mathrm{U}$ 5c 组合, 可与盐岭 Wargal 组的孢粉组合对比, Illawarra 煤系之上为 Narrabeen 群, 其底部所产孢粉组合 TR1a 与盐岭 Chhidru 组上部的孢粉组合对比 ${ }^{[17]}$. Wargal 组 下部产华南孤峰阶顶部筳类带化石 $N$. cf. margaritae, Wargal 组上部含 Clarkina asymmetrica 和 C. leveni 等吴家坪阶中下部的牙形化石. 由此可见在命名地的伊拉瓦拉极性反转的层位相 当孤峰阶顶部. 我国阳新世孤峰阶顶部相当国际年代地层表中瓜德鲁普统的沃德阶顶部.

最近在澳大利亚悉尼盆地北部确定伊拉瓦拉极性反转出现在 Wittingham 煤系 ${ }^{[18]}$, 按澳大 利亚通用年代地层表, 该煤系的时代为 Ufimian 期. 但澳大利亚的二叠系虽然采用俄罗斯乌拉 尔的传统划分方案, 两地区的生物群却属于迥然不同的生物区系, 其间缺乏可资对比的生物地 层依据, 而需要根据非生物地层资料进行校正. 实际上在乌拉尔地区, Ufimian 阶远低于伊拉 瓦拉极性反转的层位 ${ }^{[34]}$. 另一方面, 最近测得与 Wittingham 煤系下部相当的 Mulbring 组所含

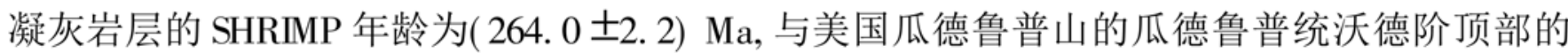
年龄接近 ${ }^{[19]}$. 后者为 $(265.3 \pm 0.2) \mathrm{Ma}$, 而且也是伊拉瓦拉极性反转层位 ${ }^{[20]}$. 这一对比进一 步证明, 命名地区的伊拉瓦拉极性反转也出现在沃德阶的顶部, 同位素年龄接近 $265 \mathrm{Ma}$.

1.3 在其他非海相沉积区的层位

在俄罗斯乌拉尔的二叠纪传统标准剖面所在地, 多年的磁性地层研究已经证明, 伊拉瓦拉 
极性反转出现在 Tatarian 阶中部, 至今未在其下方的 Kazanian 阶和 Ufimian 阶发现正极性

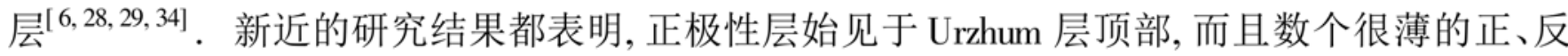
极性层交替出现, 组成不厚的磁性地层过渡带 ${ }^{[34]}$. 由此推论, 伊拉瓦拉极性反转可能不是单 一的磁性地层极性反转面.

在山西太原附近, Embleton 等人 ${ }^{[4]}$ 测得伊拉瓦拉极性反转的层位处于上石盒子组下部. 按我国传统的二叠系地层表, 石盒子组被划归上二叠统. 由于国际地质年代表 ${ }^{[21]}$ 的上二叠统 包括瓜德鲁普统和乐平统, 以致 Embleton 等误认为, 上石盒子组的下部应相当瓜德鲁普统下部 的 Ufimian 阶. 不过我国传统二叠系地层表的上二叠统与国际地质年代表的不同, 只相当于乐 平统, 并不包括瓜德鲁普统. 上石盒子组含 Gigantonoclea hallei-Fascipteris spp-Lobatannularia ensif olia 植物化石组合, 与华南龙潭组植物群相当, 时代属于瓜德鲁普统晚期至乐平世早期 ${ }^{[22]}$. 因此, 出现在石盒子组下部的伊拉瓦拉极性反转仍为瓜德鲁普世晚期 ${ }^{[23]}$.

2 地磁极性单位的综合层序

究竟晚二叠世的地磁极性转换序列应归纳为几个地磁极性地层单位为宜? 由于各剖面的 地磁极性记录的完整性和清晰程度很不相同, 目前还难以实行极性层的逐层对比, 通常根据各 时期极性层的组合特点划分为 4 个极性带.

\section{1 海相沉积区的层序}

首先, 代表伊拉瓦拉极性反转的极性过渡层及其上的 2 个层位稳定的正极性层和其间的 反极性层, 可归纳为一个正极性带 $(\mathrm{N} 1 \mathrm{P})$. 下部正极性层属于瓜德鲁普世沃德阶顶部. 其上 的反极性层和另一个正极性层与盐岭 Wargal 组下部及亚美尼亚哈契克组上部的正极性层可 以对比, 属于瓜德鲁普统卡匹敦阶( Capitanian stage). 由于这些剖面上缺乏卡匹敦阶上部和吴 家坪阶底部的沉积, 所以卡匹敦阶上部的地磁极性转换序列缺乏完整的记录, 以致该正极性层 上限的层位难以在该剖面确定. 这一缺陷可以从四川广元上寺剖面得到补充. 该处茅口组顶 部呈现反极性, 层位相当卡匹敦阶顶部的 Jinogondolella altudaensis 带. 如果这一极性层的测定 结果是可靠的, 那么 N1P 正极性带的上界即处于卡匹敦阶顶部.

瓜德鲁普统与乐平统之间的连续碳酸盐沉积目前仅见于广西来宾红水河向斜 ${ }^{[24]}$. 笔者 与 Menning ${ }^{[25]}$ 采样研究后, 因重磁化强烈, 至今未能得到可信度较高的结果. 盐岭 Wargal 组上 部含 Clarkina asymmetrica 和 C. leveni 等吴家坪阶中下部的牙形化石, 将其归属 Midian 阶 $^{[10]}$ 显 然不妥. 该部属于反极性, 表明吴家坪阶中下部应为一反极性带. 在亚美尼亚, 卡契克组上部 相当吴家坪阶的底部, 因样品稀少, 磁性记录缺失较多; 阿呼林组底部为反极性, 层位相当吴家 坪组下部 C. leveni 带, 应与盐岭 Wargal 组上部的反极性层相当.

武隆江口剖面的吴家坪组仅其下部 $50 \mathrm{~m}$ 属于吴家坪期, 基本上呈反极性. 四川广元上寺 剖面的吴家坪组仅相当吴家坪阶下部; 大隆组下部属于吴家坪阶老山亚阶, 以反极性为主. 层 11 显示反极性, 属于 Clarkina liangshanensis 带, 应归于吴家坪中下部反极性带. 层 12 至层 16 相当 $C$. liangshanensis 带上部和 $C$. orientalis 带, 为正极性层. 这一正极性层的生物地层位 置与重庆合川剖面龙潭组的正极性层 ${ }^{[26]}$ 相当, 并与盐岭 Wargal 组顶部的正极性层可以对比. 综上所述, 自卡匹敦阶顶部至吴家坪阶的 C. liangshanensis 带均以反极性为主, 是一个特征显著 的反极性带 (R1P), 而吴家坪期晚期以正极性为主, 已经进入 PTMM 的第二个正极性带 (N2P). 
李华梅等人 ${ }^{[3]}$ 将浙江梅山长兴组地磁极性转换序列划分为 4 个极性层, 即底部正极性层、 中下部反极性层、上部正极性层和顶部反极性层. 四川邻水剖面 ${ }^{[13]}$ 与之完全对应. 底部正极 性层应下延至吴家坪组顶部, 相当煤山剖面的底部正极性层. 与中下部反极性层对应的层位 实际上是由 3 个反极性层和 2 3 个正极性层构成的混合极性层. 亚美尼亚的多腊沙姆阶大 部分相当长兴阶下部, 所以多腊沙姆阶的阿呼林组正极性层大体与长兴组下部正极性层相对 应. 这样, 吴家坪阶顶部 C. orientalis 带至长兴阶底部正极性层和中下部的混合极性层均作 为PTMM的第 2 个正极性带.

长兴阶上部 Clarkina wangi 带和 C. changxingensis 带正极性层和顶部反极性层则形成 PTMM 的第二个反极性带 (R2P). 长兴组上部正极性带和顶部反极性带在四川上寺、邻水 ${ }^{[13]}$ 得 到证实. 不同的是煤山剖面最顶部为正极性层层位, 相当 C. yini 带和 C. meishanensis 带, 在 其他剖面均未发现 ${ }^{[27]}$. 由于长兴期末的沉积在世界各地的许多海相剖面均为缺失, 所以这两 个极性层也不可能普遍有记录. 另一方面, 三叠纪最底部的沉积却广泛发育, 测得的地磁极性 则一致为正向. 因此, 长兴组上部正极性层和顶部反极性层的记录虽然不多, 却相当特征, 应 划分出来作为 PTMM 的第 2 个反极性带.

\section{2 非海相沉积区的序列}

俄罗斯乌拉尔的二叠系传统标准剖面经长期研究, 极性变换的序列已基本确立. Khramov $^{[6]}$ 将其划分为 4 个正极性带和 1 个反极性带, Molostovsky 等人 ${ }^{[28]}$ 则归纳为 2 个正极性 带和 2 个反极性带. 后者的极性带与本文归纳的结果很相近. N1P 带包括 Tatarian 阶 Urzhum 层顶部的极性过渡层, Sverodvinsk 层的下部 3 个地层跨度较长的正极性带和 2 个短的反极性 带, 层位约略与瓜德鲁普统上部正极性带相当. Sverodvinsk 层上部主要呈现反极性, 仅含有一 个短暂的正极性层, 被归入 R1P 带. 此带出现的层位和极性层结构与吴家坪阶中、下部反极 性带相对应. Tatarian 阶 Vyatsk 层下部属于 N2P 带, 以正极性层为主, 间夹 2 个短的反极性层, Khramov $^{[29]}$ 将其划分为 2 个正极性带 N2P 和 N3P. 此带相当吴家坪阶上部至长兴阶中下部正 极性带. Vyatsk 层亚阶上部包含地层跨度相近的 2 个反极性层和一个正极性层, 归属 R2P 带, 但 Khramov ${ }^{[29]}$ 称 N4P 带. 此带的层位与长兴阶上部的正极性带相当. 值得注意的是在传统标 准剖面上, 二叠系-三叠系界线上下分别为正极性层和反极性层, 与大多数海相剖面的层序一致.

我国太原附近的晚二叠世的上石盒子组和孙家沟组包含 13 个极性层 ${ }^{[4]}$. 上石盒子组下 部的 2 个正极性层及其间的反极性层与瓜德鲁普统上部的极性带相当. 此组的中、上部以反 极性层为主, 与吴家坪阶中下部一致. 上石盒子组顶部及孙家沟组以正极性为主, 应当与吴家 坪阶上部和长兴阶相当, 不过仅包括 3 个正极性层和 2 个反极性层, 难以与海相剖面的极性地 层序列详细对比, 可能系长兴期晚期沉积不甚完整之故.

澳大利亚悉尼盆地和 Gunnedah 盆地晚二叠世煤系的磁性地层复合序列包含的极性层较 多, 共有近 20 多层 ${ }^{[18]}$. 这可能是 Gunnedah 盆地的 Wittingham 煤系厚达 $3000 \mathrm{~m}$, 所以极性变换 的记录比其他剖面更为完整, 以致 Wittingham 煤系中、上部虽以反极性为主, 但夹有 5 个较薄 的正极性层. 如果将这一反正极性与吴家坪阶中、下部的反极性带 (R1P) 对比, 那么 Wittingham 煤系下部的 2 个正极性层即属于瓜德鲁普上部正极性带(N1P), 而 Wittingham 煤系顶部和 Black Jack 组或 Illawarra 煤系以正极性为主的层位应相当吴家坪阶上部和长兴阶 ( N2P 和 $\mathrm{R} 2 \mathrm{P})$. 
Rotliegendes 统上部的 Havel 组和 Elbe 组以正极性为主, 间夹 4 个反极性层, 大体相当瓜德

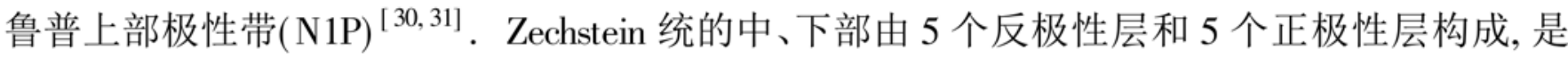
整个晚二叠世磁性剖面中反极性跨度较大的一段, 可与吴家坪阶中、下部 R1P 带对比. Zechstein 统上部以正极性为主, 应包含吴家坪阶上部和部分长兴阶的极性层.

以上对比说明,在东欧、西欧、亚洲和澳大利亚的非海相地层的晚二叠世磁性剖面，与以 海相地层为基础建立的综合层序可以实行磁性带的对比. 互相印证的结果说明, 瓜德鲁普世 晚期正极性带和吴家坪期反极性带包含的极性层的数目应比海相剖面记录的多. 因此, 复合 极性层序的 N1P 带和 R1P 带分别采用乌拉尔 Tatarian 阶中下部和悉尼盆地 Wittingham 煤系的 极性变换序列作为代表.

\section{3 国际对比意义}

磁性地层序列为国际上二叠统对比的关键问题提供了便捷、可靠的解决途径. 根据磁性 地层序列, 瓜德鲁普统和乐平统上部的标准层序与远离标准剖面的同期地层之间大体可以进 行阶一级的对比. 如此高精度的全球对比是单纯使用生物地层方法难以达到的. 由于新的对 比方案与传统的观点分歧较大, 所以又引发出富有挑战性的新问题. 长期以来, 传统标准的 Tatarian 阶一直被认为相当新标准的乐平统. Ufimian 阶和 Kazanian 阶相当瓜德鲁普统. 磁性地 层对比则证明, 只有 Tatarian 阶的 Sverodvinskian 层上部和 Vyaskian 层相当乐平统; Sverodvinskian 层下部相当瓜德鲁普统的沃德阶顶部和卡匹敦阶. 由此推论, Tatarian 阶下部的 Urzhum 层与 Kazanian 阶、Ufimian 阶只相当于瓜德鲁普统的 Roadian 阶和 Wordian 阶, 与传统对比方案 差别较大. 这是有待进一步依据同位素年龄资料证实的问题. 更加令人费解的是南北大陆二 叠系的对比问题. 澳大利亚以及冈瓦纳大陆其他地区的二叠系习惯采用二叠系传统标准层序 进行划分, 而南半球冈瓦纳大陆和北半球安加拉大陆两大古地理区的二叠系, 特别是上二叠统 的对比却一直缺乏实际依据 ${ }^{[32]}$. 磁性对比表明冈瓦纳大陆的所谓 Ufimian 期地层与传统标准 层序的 Ufimian 阶并不同期, 而只相当于 Tatarian 阶的中部, 或国际新标准层序的瓜德鲁普统上 部 ${ }^{[33]}$. 这种修改冈瓦纳大陆二叠纪年代地层格架的趋势得到同位素年龄资料的支持. 一旦 冈瓦纳大陆的“Ufimian 阶” 需要修正, 南北二叠系对比的基本格局都将随之大幅度调动. 这无 疑是一个涉及范围更广的地层问题.

致谢此项研究得到中国科学院资源与生态环境研究项目(编号: $\left.\mathrm{K}_{2} 951-\mathrm{B}_{1}-409\right)$ 和国家自然科学基金( 批准 号: 49672092) 资助项目.

\section{参 考 文 献}

1 Jin Yugan, Wardlaw B R, Glenister B F, et al. Permian chronostratigraphic subdivisions. Episodes, 1997, 20( 1): 10 15

2 Menning M. A rivised permian polarity time scale. EUG VII Abstract, Strasbourg: 1993. 4 8

3 李华梅, 王俊达. 浙江长兴煤山二叠-三叠系界线剖面磁性地层特征. 中国科学, 1989, B( 6)：652 658

4 Embleton B J J, McElhinny M W, Ma X H, et al. PermorTriassic magnetostratigraphy in China: the type section near Taiyuan, Shanxi Province, North China. Geophys J Int, 1996, 126: 382 388

5 Irving, E, Parry L G. The magnetism of some permian rocks from new south wales. Geophysical Journal of the Royal Astronomical Society, 1963, 7: 395 411

6 Khramov A N. Palaeomagnetic investigations of Upper Permian and Lower Triassic sections on the northern and eastern Russian Platform. Trudy VNIGRI, Leningrad: Nedra, 1974, 204: 145 174 
7 Peterson D N, Nairn A E M. Palaeomagnetism of Permian red beds from the southrwestern United States. Geophys J Roy Astron Soc, 1971, 23: 191 207

8 Menning M. Fortschritte des Palaomagnetismus im Perm Mitteleuropas. Z geol Wiss, 1981, 9( 11): 1247 1252

9 Kotlyar G V, Komissarova R A, Khramov A N, et al. Paleomagnetism of Upper Permian rocks of Transcaucasia. Dokl Akad Nauk SSSR, 1984, 276: 669 674

10 Haag M, Heller F. Late Permian to Early Triassic magnetostratigraphy. Earth Planet Sci Lett, 1991, 107: 42 54

11 Wardlaw B R, Pogue K R. The Permian of Pakistan. In: Scholle P A, Peryt T M, Ulmer-Scholle D S eds. The Permian of Northern Pangea, Volume 2. Sedimentary Basins and Economic Resourece, 1995. 215 224

12 陈海泓, 孙 枢, 李继亮, 等. 四川武隆地区二叠一三叠系磁性地层学研究. 中国科学, B 辑, 1992, 12: 1317 1324

13 陈海泓, Heller F．四川凉桥晚二叠世磁性地层学剖面．科学通报，1994，39(1)：70 72

14 Heller F, Chen H H, Dobson J, et al. PermiarrTriassic magnetostratigraphy-new results from South China. Phys Earth Planet Int, 1995, 89: 281 295 $1 \sim 23$

16 Bowring S A, Erwin D H, Jin Y G, et al. U/ Pb Zircon Geochronology and Tempo of the EndPermian Mass Extinction. Science, 1998, 280 ( 5 366) : 1039 1045

17 Balme B E. Palynology of Permian and Triassic strata in the Salt Range and Surghar Range, West Pakistan. In: Kummel B, Teichert C eds. Stratigraphic Boundary Problem: Permian and Triassic of West Pakistan. The Univ Press Kansas, 1970. 305 405

18 Theveniaut H, Klootwijik C, Giddings J. Magnetostratigraphy of the Late Permian coal measures, Sydney \& Gunnedah Basin: A regional \& global correlation tool. Proc. 28th Newcastle Symposium on “Advance in the study of the Sydney Basin”, 15 17 April, 1994, Univ. of Newcastle press, Newcastle, New Soutt Wales, 1994. 11 23

Roberts, J, ClaourL Long J C, Foster C B. SHRIMP zircon dating of the Permian System of eastern Australia. Australian J Earth Sci, 1996, 43: 401 421

Glenister B F. Wardlaw B R. Permian Chronostratioranhic subdivisions. Permonhiles. 1998. 31: 35 36

Harland W B, Armstrong R L, Cox A V, et al. A geological Time Scale. Camophiles: Cambridge University Press, $1990.1 \sim 263$.

Sheng J Z, Jin Y G. Correlation of Permian deposits in China. Nanjing University Press, Nanjing. Palaeoworld, 1994, 4: 14 113

Menning M, Jin Yugan. Comment on "PermorTriassic magnetostratigraphy in China: the type section near Taiyuan, Shanxi Province, North China”. by Embleton B J J, McElhinny M W, Ma X H, et al eds. Geophys J Int, 1998, 133: 213 216 Jin Yugan, Mei Shilong, Zhu Zili. The MaokouarrLopingian boundary sequences in South China. Palaeoworld, 1994, 1( 4) : 119 132

Menning M, Jin Yugan, Shen Shuzhong. The Illawarra Reversal (265 Ma) in the marine Permian, Guangxi, South China. Abstr 30th Int Geol Congr, Beijing, 1996, 2: 9 Jour Geophys Res, 1989, 94: 7343 7363

Wardlaw B R, Mei S L. Clarkina (Conodont) Zonation for the upper Permian of China. Permophiles, 1998, 31: 3 5

28 Molostovsky E A. Paleomagnetic stratigraphy of the Permian System. Intern Geol Rev, 1992, 34: 1001 1007

Khramov A N, Goncharov G I, Komissarova R A, et al. Palaeomagnetology. Leningrad: I2d Nedra, 1982. 1 312

Dachroth W. Gesteinsmagnetische Marken im Perm Mitteleuropas. Geol Jahrb. Hannover E, 1976, 10: 3 63

31 Menning M, Katzung G, Lutzner H. Magnetostratigraphic investigations in the Rotliegendes ( $300 \sim 252$ Ma) of Central Europe. Zeitschrift fir Geologischen Wissenschaften, 1988, 16: 1045 1063

Archbold N W, Dickins J M. Australian Phanerozoic Timescales: 6. A standard for the Permian System in Australia. Bureau of Mineral Resources, Australia Record, 1991, 36: 1 17

33 Jin Yugan, Menning M. A possible north south correlation of the Permian. Permophiles, 1996, 29: 40 42

34 Burov B V, Nugaliev D K, Heeler F. Problems of palaeomagnetostratigraphic correlation between the Upper Permian stratotype and the Tethyan marine formations. In: Sevelev A I et al eds. Permian deposits of Republic Tatarstan. I2d Kazan Univ: Kazan, 1996. 93 99 (1998-05-25 收稿, 1998-10-08 收修改稿) 